\title{
Effect of different mycobionts on symbiotic germination and seedling growth of Dendrobium officinale, an important medicinal orchid
}

\author{
Ying Zhang ${ }^{1}$, Yuan-Yuan Li ${ }^{1}$, Xiao-Mei Chen ${ }^{1}$, Shun-Xing Guo ${ }^{1 *}$ and Yung-I Lee ${ }^{2,3^{*}}$ (1)
}

\begin{abstract}
Background: Orchids maintain a symbiotic relationship with mycorrhizal fungi in the lifecycle. Previous reports indicated that diverse mycobionts may have different roles during orchid growth and development. Although various mycorrhizal fungi have been isolated from Dendrobium roots and protocorms, little is known about their specific effects on seed germination and seedling growth. To understand the specific role of isolated fungal strains (i.e., Tulasnella and Sebacina), we used symbiotic culture to compare the effect of 6 fungal strains on seed germination and seedling growth of Dendrobium officinale, an important Chinese medicinal orchid.

Results: In symbiotic germination tests, 6 fungal strains (4 Tulasnella strains and 2 Sebacina strains) promoted seed germination with different efficiencies. Seeds inoculated with Tulasnella strains S6 and S7 conferred higher germination percentage and faster protocorm development than other fungal strains. In symbiotic cultures, seedlings inoculated with Sebacina strain S3 had optimal fresh and dry matter yield. Also, Tulasnella strains S6 and S7 promoted seedling growth with good fresh and dry matter yield. Sebacina strain S2 inoculation greatly enhanced root and tiller production and the content of total crude polysaccharides, although seedlings were smaller with less fresh and dry matter yield than other seedlings.
\end{abstract}

Conclusions: Tulasnella and Sebacina strains could promote seed germination and seedling growth of $D$. officinale with different efficiencies. Our results suggest a non-specific mycorrhizal association and development-dependent preference. Our data provide the basic knowledge for use of different fungal strains in conservation and/or production practices of $D$. officinale.

Keywords: Dendrobium, Mycorrhiza, Seed germination, Seedling growth, Crude polysaccharides

\section{Background}

A common example of widespread mutualism is the association of mycorrhiza and land plants, whereby fungi retrieve mineral nutrition in the soil and pass it back

\footnotetext{
*Correspondence: sxguo1986@163.com; leeyungi@hotmail.com

${ }^{1}$ Institute of Medicinal Plant Development, Chinese Academy of Medical

Sciences \& Peking Union Medical College, Beijing 100193, People's

Republic of China

2 Biology Department, National Museum of Natural Science,

Taichung 40453, Taiwan

Full list of author information is available at the end of the article
}

to plants and plants contribute the products of photosynthesis to fungi (Smith and Read 2008). In orchids, a compatible mycorrhizal association is a requirement for seed germination under natural conditions (Rasmussen 1995). During seed germination and early growth of protocorms, mineral nutrients are transferred from fungi to the orchid, a phenomenon known as "mycoheterotrophy" (Leake 1994). After successful infection, fungal hyphae coil and form pelotons within the cortical cells (Smith and Read 2008). Afterward, the pelotons senesce and collapse to release nutrients in orchid cells (Kuga et al. 
2014). The formation and degradation of pelotons play a key role in the exchange and absorption of nutrients between an orchid and its mycorrhizal fungus (Dearnaley and Cameron 2017; Fochi et al. 2017).

Most green orchids form mycorrhizae with polyphyletic Rhizoctonia-like fungi, including Tulasnella, Ceratobasidium, Thanatephorus and Sebacina clade B (Dearnaley et al. 2012). Orchids may have high specificity in their fungal partners; an example is mycoheterotrophic orchids (Leake 1994; Bidartondo 2005) such as Corallorhiza striata complex (Barrett et al. 2010) and Hexalectris (Kennedy et al. 2011). In contrast, other orchids may associate with diverse sets of fungal partners; for example, Cypripedium californicum associates with Tulasnellaceae, Ceratobasidiaceae, and Sebacinales (Shefferson et al. 2007). Moreover, Tulasnellaceae, Thelephoraceae, Ceratobasidiaceae, Sebacinales, Russulaceae and Clavulinaceae were detected in Cymbidium goeringii and Cymbidium lancifolium (Ogura-Tsujita et al. 2012). Also, a succession of fungal colonization over the orchid life cycle occurs; for example, Gastrodia elata, a mycoheterotrophic orchid, needs Mycena for seed germination, but subsequent colonization by Armillaria is required for the orchid's further development (Xu and $\mathrm{Mu}$ 1990). Although orchids are colonized by different fungi, not all colonized fungi have the same effect on the growth and development of orchids. In Vanilla, different mycorrhizal fungal isolates from roots had dissimilar effects on growth and survival (Porras-Alfaro and Bayman, 2007). In Dendrobium, Tulasnella isolated from $D$. aphyllum protocorms could stimulate germination, but Trichoderma could not improve germination ( $\mathrm{Zi}$ et al. 2014).

Dendrobium officinale, one of the most important Dendrobium species in China, has long been used in traditional Chinese medicine to treat chronic diseases (Pharmacopoeia Committee of the P. R. China 2005). Previous investigations showed various fungal mycobionts identified in and/or isolated from roots or protocorms of medicinal Dendrobium species (Chen et al. 2012; Tan et al. 2014; Zi et al. 2014; Wang et al. 2017), which suggests no high specificity in the fungal association. However, the roles of various fungal strains isolated from Dendrobium species have never been tested systematically with controlled in vitro culture methods.

To understand the effects of various fungal strains on ontogenetic stages in orchid, we compared the effect of 6 fungal strains of Tulasnella and Sebacina on symbiotic germination and tested their ability to promote seedling growth by examining the growth rate and crude polysaccharides content. Knowledge of mycorrhizal association in medicinal Dendrobium species would be helpful for propagation, commercial cultivation and conservation.

\section{Methods}

\section{Plant materials}

Plants of $D$. officinale were maintained in a plantation in Jinhua, Zhejiang, China. At the time of anthesis, in March, flowers were hand-pollinated, and mature capsules were collected just before dehiscence, in November, for the following experiments. In total, 10 capsules were collected for experiments.

\section{Fungal strains for symbiotic cultures}

In our laboratory, we have developed efficient methods for fungal isolation from $D$. officinale protocorms and roots (Li et al. 2018a). In this study, Sebacina strains S2 and S3 were isolated from mycorrhizal protocorms by the technique of in situ seed baiting in the natural habitat in Yunnan Province of Southwestern China, and Tulasnella strains S4, S5, S6 and S7 were isolated from roots of mature plants in the same area. These fungal strains were maintained in our laboratory and deposited at the microbiological center of the Institute of Medicinal Plant Development, Chinese Academy of Medical Sciences \& Peking Union Medical College, Beijing (see Additional file 1: Table S1). Before symbiotic culture, fungal strains were inoculated on potato dextrose agar medium (potato $200 \mathrm{~g} \mathrm{~L}^{-1}$, glucose $20 \mathrm{~g} \mathrm{~L}^{-1}$, agar $12 \mathrm{~g} \mathrm{~L}^{-1}, \mathrm{pH} 5.2$ before autoclaving) according to the procedure by $\mathrm{Li}$ et al. (2018b) in darkness at $25 \pm 1{ }^{\circ} \mathrm{C}$ for 7 days. The actively growing mycelia from the colony margin were used as the fungal inoculum in subsequent symbiotic cultures.

\section{Molecular identification and phylogenetic analysis}

DNA was extracted from pure mycelium cultures of fungi by using the Rapid Plant Genomic DNA Isolation Kit (Sangon Biotech, Shanghai) according to the manufacturer's instructions. The internal transcribed spacer (ITS) region of the fungal nuclear rRNA gene was amplified with the primer pairs ITS1F/ITS4R (ITS1F: $5^{\prime}$-TCC GTAGGTGAACCTGCGG-3'; ITS1R: 5'-TCCTCCGCT TATTGATATGC-3') (White et al. 1990; Gardes and Bruns 1993). The PCR amplification was initial denaturing at $95^{\circ} \mathrm{C}$ for $5 \mathrm{~min}$, followed by 35 cycles of denaturing at $95^{\circ} \mathrm{C}$ for $1 \mathrm{~min}$ each, annealing at $55^{\circ} \mathrm{C}$ for $1 \mathrm{~min}$, extension at $72{ }^{\circ} \mathrm{C}$ for $1 \mathrm{~min}$, and final extension for $5 \mathrm{~min}$ at $72{ }^{\circ} \mathrm{C}$. Sequences were identified by a BLAST search against the NCBI sequence database (GenBank). For phylogenetic analysis, other ITS sequences of Tulasnella and Sebacina from various orchids were obtained from GenBank. The sequence of Armillaria sinapina was used as the outgroup. DNA sequences were aligned by using the ClustalW algorithm in MEGA 7.01, followed by manual adjustment. Distance trees were obtained by using the neighbor-joining (NJ) method (Saitou and Nei 1987) with a Tajima-Nei method. For assessing relative robustness 
for branches, the bootstrap method was used with 1000 replicates (Felsenstein 1985).

\section{Symbiotic germination}

Before sowing seeds, oat meal agar medium (OMA: oat $4 \mathrm{~g} \mathrm{~L}^{-1}$, agar $8 \mathrm{~g} \mathrm{~L}^{-1}, \mathrm{pH} 5.2$ before autoclaving) placed in a 9-cm diameter Petri dish was inoculated with 4 pieces of fungal inoculum for each fungal strain, then Petri dishes were placed in darkness at $25 \pm 1{ }^{\circ} \mathrm{C}$ for 7 days as described by Li et al. (2018a, b). Capsules were rinsed under tap water for $10 \mathrm{~min}$ and surface-sterilized with $75 \%$ ethanol for $60 \mathrm{~s}$, followed by immersion in $2.5 \%$ sodium hypochlorite solution for $15 \mathrm{~min}$. After rinsing 3 times with sterile water, capsules were cut and opened to remove seeds. About 200 seeds were sown onto the surface of OMA medium in each Petri dish. 12 replicates (Petri dishes) were used for each fungal treatment. Petri dishes without fungal inoculum were the control. After inoculation, the exact number of seeds of each dish was counted under a stereomicroscope. The cultures were placed in a growth room under a 12/12-h light/dark photoperiod at $30 \mu \mathrm{mol} \mathrm{m}{ }^{-2} \mathrm{~s}^{-1}$ (daylight fluorescent tubes $\mathrm{FL}-20 \mathrm{D} / 18,20 \mathrm{~W})$ at $25 \pm 1{ }^{\circ} \mathrm{C}$.

\section{Symbiotic culture of seedlings}

To compare the effect of different fungal strains on seedling growth in symbiotic culture, young seedlings were obtained from asymbiotic culture before inoculation. Mature seeds were removed from capsules and placed onto modified Murashige and Skoog medium (Murashige and Skoog 1962), containing half-strength macroelements with full-strength microelements, vitamins, and amino acids and supplemented with $20 \mathrm{~g} \mathrm{~L}^{-1}$ potato homogenate, and $20 \mathrm{~g} \mathrm{~L}^{-1}$ sucrose, and solidified with $7 \mathrm{~g} \mathrm{~L}^{-1}$ agar. The $\mathrm{pH}$ of the medium was adjusted to 5.7 with $1 \mathrm{~N} \mathrm{NaOH}$ solution before autoclaving at $101.33 \mathrm{kPa}$ and $121{ }^{\circ} \mathrm{C}$ for $20 \mathrm{~min}$. The cultures were placed in the growth room under a $12 / 12$-h photoperiod at $25 \pm 1{ }^{\circ} \mathrm{C}$ as described previously. After 6 months of sowing, seedlings about $3 \mathrm{~cm}$ tall were selected for symbiotic culture.

For symbiotic culture of seedlings, OMA medium combined with $\mathrm{H} 1$ basal salts $\left(200 \mathrm{mg} \mathrm{L}{ }^{-1} \mathrm{Ca}\left(\mathrm{NO}_{3}\right)_{2} \cdot 4 \mathrm{H}_{2} \mathrm{O}\right.$, $100 \mathrm{mg} \mathrm{L}^{-1} \mathrm{KCl}, 200 \mathrm{mg} \mathrm{L}{ }^{-1} \mathrm{KH}_{2} \mathrm{PO}_{4}, 100 \mathrm{mg} \mathrm{L}^{-1}$ $\mathrm{MgSO}_{4} \cdot 7 \mathrm{H}_{2} \mathrm{O}, 100 \mathrm{mg} \mathrm{L}^{-1}$ yeast extract, $2 \mathrm{~g} \mathrm{~L}^{-1}$ sucrose) described by Rasmussen (1995) was used. Each glass bottle $(9 \mathrm{~cm}$ diameter, $12.5 \mathrm{~cm}$ tall) contained $125 \mathrm{~mL}$ culture medium, and $\mathrm{pH}$ was adjusted to 5.7 before autoclaving. Before symbiotic cultures of seedlings, each glass bottle was inoculated with 4 pieces $\left(0.5 \mathrm{~cm}^{3}\right)$ of fungal inoculum. As the hyphae had spread over the surface of culture medium, uniform seedlings ( $450 \mathrm{mg}$ fresh weight, single shoot of $3 \mathrm{~cm}$ tall with 5 roots) from asymbiotic cultures were selected and transferred to glass bottles; 12 replicates (glass bottles) were used, and each glass bottle contained 6 seedlings for each fungal treatment. After 3 months of symbiotic culture, the fresh weight, dry weight, shoot number and root number of each seedling were recorded. Glass bottles without fungal inoculum were the control. Symbiotic cultures were placed in the growth room under a $12 / 12$-h photoperiod at $25 \pm 1{ }^{\circ} \mathrm{C}$ as described previously.

\section{Histological and histochemical observations}

Developing mycorrhizal protocorms were fixed in $1 \%$ glutaraldehyde in $0.1 \mathrm{M}$ phosphate buffer $(\mathrm{pH}$ 6.8) for $4 \mathrm{~h}$ at room temperature and dehydrated with an ethanol series, then embedded in Technovit 7100 resin (Kulzer and Co., Wertheim, Germany) according to Yeung and Chan (2015). Serial, 3- $\mu$ m-thick sections were cut and stained with Periodic acid-Schiff reaction for total insoluble carbohydrates, then counterstained with $0.05 \%(\mathrm{w} / \mathrm{v})$ toluidine blue $\mathrm{O}$ (TBO) for general histology. Sections were observed under a light microscope (Axio ImagerA1, Carl Zeiss AG) and the images were captured digitally by using a CCD camera. For fungal hyphae staining, mycorrhizal protocorms were fixed as described above. Subsequently, samples were washed with $1 \times$ PBS ( $\mathrm{pH}$ 7.4) for 3 times for $10 \mathrm{~min}$, then incubated at room temperature for $90 \mathrm{~min}$ in $1 \times$ PBS containing the chitin-specific dye WGA-FITC at $10 \mu \mathrm{g} \mathrm{L}^{-1}$ (Molecular Probes, Karlsruhe, Germany). After a washing with $1 \mathrm{x}$ PBS for 3 times for $10 \mathrm{~min}$, samples were mounted on glass slides and observed under a confocal microscope (LSM510, Carl Zeiss, Germany) with a 488-nm laser line and detected at 505-540 nm.

\section{Measurement of polysaccharides content}

Polysaccharides were extracted as described (Wang et al. 2018) with minor modification. Seedling stems were dried in an oven at $80{ }^{\circ} \mathrm{C}$ for $24 \mathrm{~h}$. Dried stems were ground into a fine powder and sieved through a 40-mesh sieve. Each ground sample of $20 \mathrm{mg}$ was placed into a $2-\mathrm{mL}$ centrifuge tube with $440 \mu \mathrm{L}$ distilled water for about $2 \mathrm{~h}$, then extraction was performed at $63^{\circ} \mathrm{C}$ for $18 \mathrm{~min}$ in a KH5200DE ultrasonic instrument (Kunshan Hechuang Ultrasonic Machinery Co., Jiangsu, China). The extraction process was repeated 3 times, and supernatants were combined. The water extract was precipitated with 4volumes of absolute ethanol, kept at $4{ }^{\circ} \mathrm{C}$ for $24 \mathrm{~h}$, then centrifuged at $5180 \times g$ at $4{ }^{\circ} \mathrm{C}$ for $20 \mathrm{~min}$. The precipitate was precipitated in $80 \%$ ethanol, then dissolved in $1 \mathrm{~mL}$ water. After mixing, $100 \mu \mathrm{L}$ polysaccharide aqueous solution and $1.9 \mathrm{~mL}$ water were added into a plugged test tube, then $1 \mathrm{~mL}$ of $5 \%$ phenol solution and $5 \mathrm{~mL}$ concentrated sulfuric acid were successively added to the same test tube. The test tube was placed into a 
boiling water bath for 20 min. After cooling, colorimetric determination was measured at $490 \mathrm{~nm}$ by using the EnSpire ${ }^{\circledR}$ Multimode Plate Reader (PerkinElmer, USA). Standard glucose at $99.9 \%$ (CAS: 50-99-7) was purchased from the National Institutes for Food and Drug Control (Beijing), with glucose solutions $(0,12.5,25,50,100$ and $150 \mu \mathrm{g} \mathrm{mL}^{-1}$ ) as standards. The linear regression equation was $y=6.9023 x+0.0824\left(R^{2}=0.9993\right)$. The content of polysaccharides in each sample $(\mathrm{Y})$ was calculated as follows:

$$
\mathrm{Y}=\frac{\mathrm{Cs} \times \mathrm{V} \times \mathrm{D}}{\mathrm{M}} \times 100
$$

where $\mathrm{Y}$ is the content of crude polysaccharides $(\mathrm{mg} / 100 \mathrm{mg}), \mathrm{Cs}$ is the concentration of glucose in the tested sample solution $\left(\mu \mathrm{g} \mathrm{mL} L^{-1}\right), \mathrm{V}$ is volume of the tested sample solution $(\mathrm{mL}), \mathrm{D}$ is the dilution multiple of sample solution, and $\mathrm{M}$ is the sample weight $(\mathrm{mg})$.

\section{Experimental design and data analysis}

In symbiotic germination experiments, each Petri dish was observed and recorded under a stereomicroscope every 2 weeks after sowing. Germination and developmental stages of protocorms were defined and scored according to Stewart et al. (2003). Germination was defined as emergence of the embryo from the seed coat. All experiments (i.e., symbiotic cultures of seed germination and seedlings) were arranged in a completely randomized design. The data were analyzed by using one-way analysis of variance (ANOVA). Mean separation was performed by Fisher's protected least significant difference test $(P<0.05)$ using SPSS 22.0 (IBM, Chicago, IL, USA).

\section{Results}

\section{The effect of fungal strains on symbiotic germination}

The results of phylogenetic analysis indicated that 6 isolated Rhizoctonia-like fungal strains were clustered with Tulasnellaceae and Sebacinales (Fig. 1, Additional file 1: Table S1). According to molecular and morphological data, 4 Tulasnella and 2 Sebacina strains were identified. The cultures of Tulasnella strains S4, S5, S6 and S7 showed creamy white colonies, whereas those of Sebacina strains $\mathrm{S} 2$ and S3 exhibited yellowish white colonies. With the fungal strains S3, S6 and S7, growth was rapid on potato dextrose agar medium at $25 \pm 1{ }^{\circ} \mathrm{C}$, but with strains S2, S4 and S5, growth was slower (Additional file 2: Figure S1).

In the symbiotic germination experiments, the 4 Tulasnella and 2 Sebacina strains could induce seed germination (Fig. 2a). After 1 week of inoculation, embryos had become swollen, and fungal hyphae congregated at the suspensor end of embryos (Fig. 2b). At this stage, minor seed coat rupture was observed. After

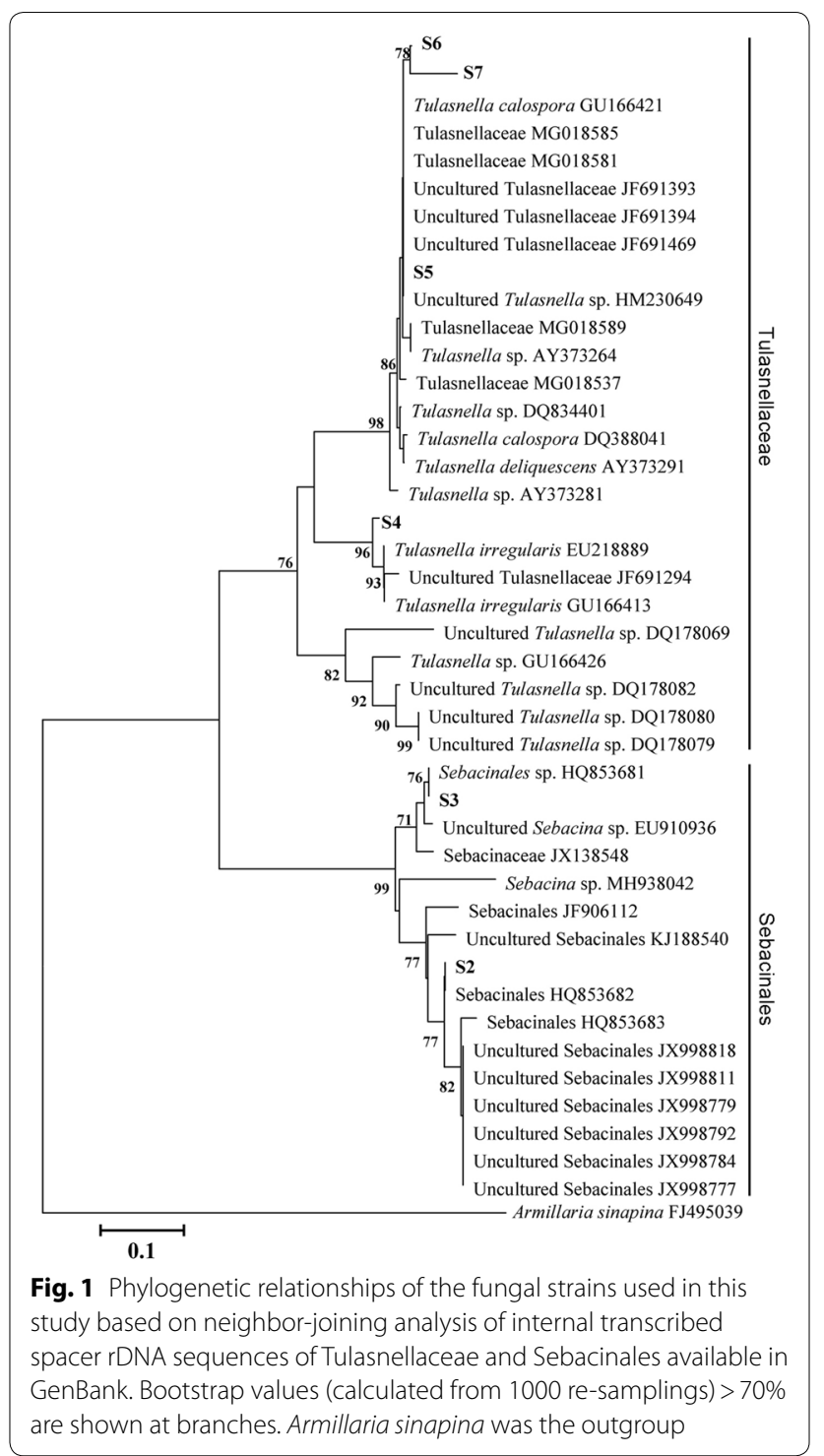

2 weeks of inoculation, embryos continued to enlarge, which resulted in a major rupture of the seed coat (Fig. 2c). Fungal hyphae had colonized primarily in the outer and inner cells at the basal part (the suspensor end) of protocorms and formed the intracellular pelotons (Fig. 2c).

The 6 fungal strains had the potential to promote seed germination at different efficiencies (Fig. 3) and to different developing protocorm stages (Table 1, Additional file 3: Figure S2). In the control (asymbiotic OMA), seeds became swollen, with rupture of the seed coat (stage 2), but no further embryo development occurred. After 5 weeks of inoculation, seeds inoculated with Tulasnella strains S6 and S7 showed higher germination rate than the other fungal strains (Table 1). However, by 9 weeks of inoculation, seeds inoculated 

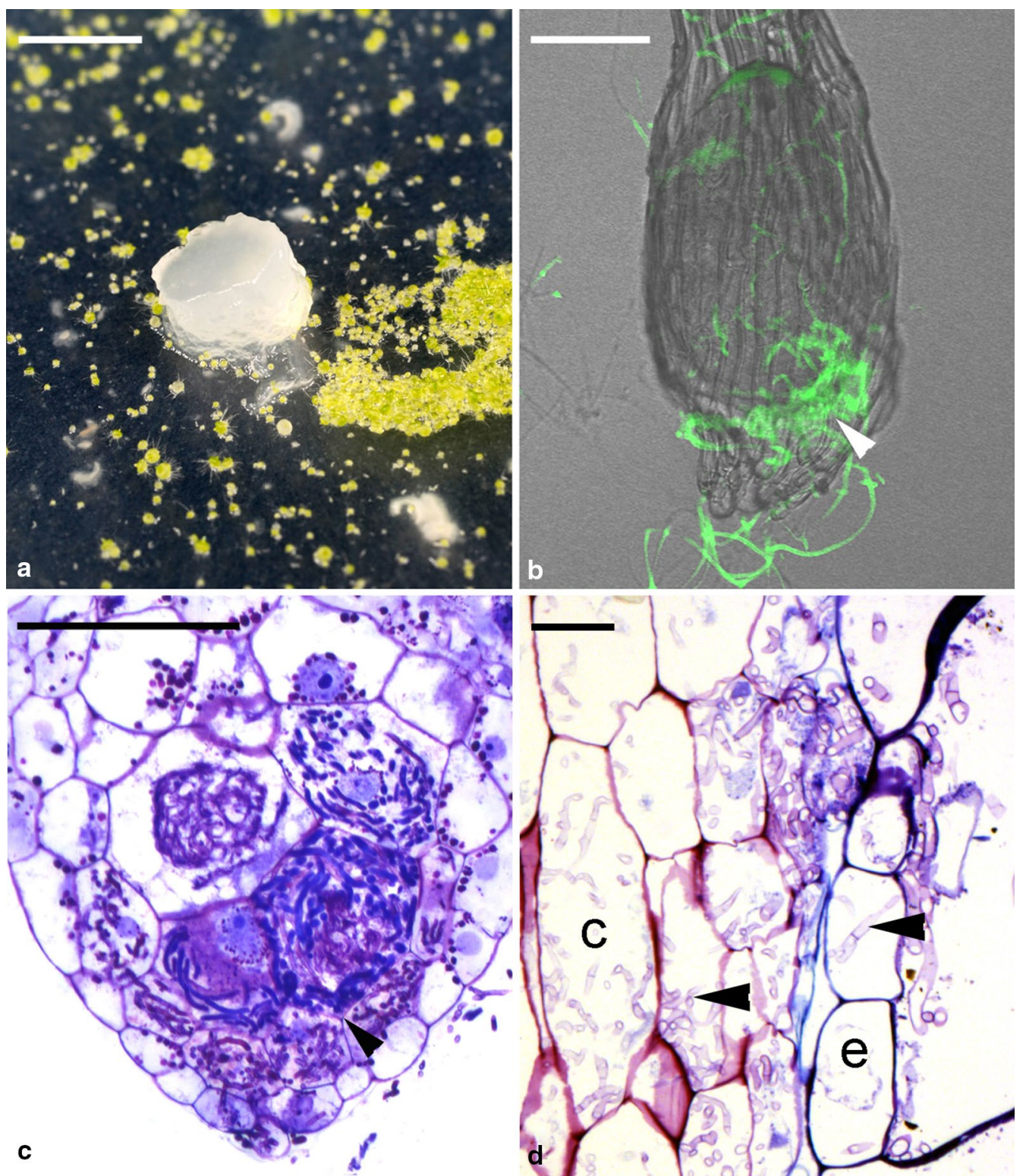

Fig. 2 Symbiotic cultures of D. officinale seeds and seedlings. a Symbiotic seed germination of D. officinale seeds. Protocorms ruptured the seed coats and turned green. Scale bar $=10 \mathrm{~mm}$. b Fungal staining using WGA-FITC (green) showing fungal hyphae (arrowhead) aggregated and penetrating the suspensor end of embryo. Bar $=40 \mu \mathrm{m}$. c Longitudinal section of a developing protocorm showing fungal hyphae (arrowhead) colonizing the basal cortical cells of an enlarged embryo. Scale bar $=50 \mu \mathrm{m}$. $\mathbf{d}$ Longitudinal section of a seedling root showing fungal hyphae (arrowheads) colonizing the epidermis (e) and cortical cells (c). Scale bar $=15 \mu \mathrm{m}$

with $\mathrm{S} 7$ had more protocorms reach stage 5 than those inoculated with S6 (Table 1). Seeds inoculated with Sebacina strains S2 and S3 and Tulasnella strain S4 showed a similar germination rate. In contrast, seeds inoculated with Tulasnella strain S5 had a slower germination rate than the other fungal strains, and the development of protocorms reached only stage 3 by 15 weeks of inoculation.

\section{The effect of fungal strains on seedling growth} in symbiotic cultures

Light microscopy revealed that all fungal strains formed a symbiotic association with $D$. officinale seedlings, as evidenced by the presence of pelotons in the cortical region of roots (Fig. 2d). Growth of D. officinale seedlings differed greatly with the fungal inoculations (Fig. 4). Seedlings inoculated with Sebacina strain S3 showed optimal 


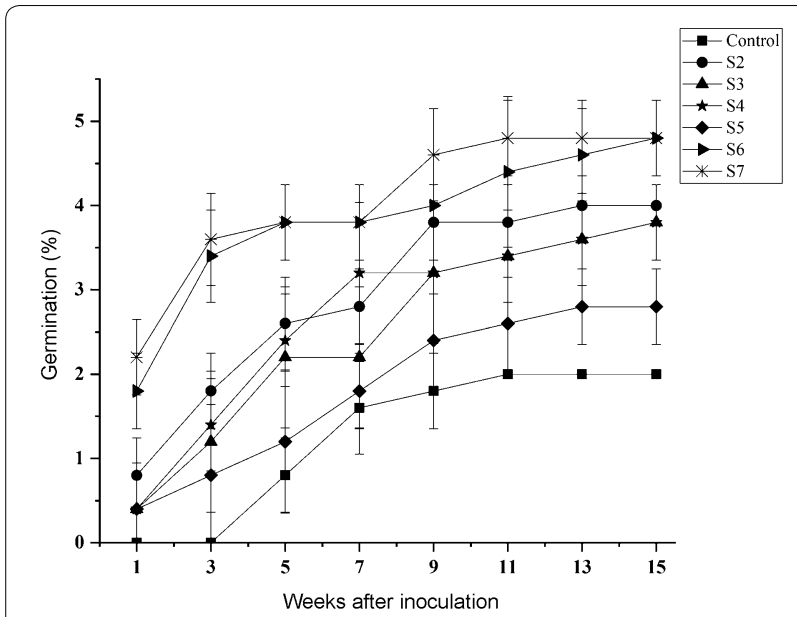

Fig. 3 Germination rate of D. officinale seeds inoculated with different fungal strains. After 1 week of inoculation, germination was recorded for each treatment every 2 weeks. Data are mean \pm SE $(n=3)$

fresh and dry matter yield. Also, seedlings inoculated with Tulasnella strains S6 or S7 showed good fresh and dry matter yield (Fig. 4). Number of roots and tillers produced was significantly greater with Sebacina strain S2 inoculation, although the seedlings were smaller and accumulated relatively little fresh and dry matter (Fig. 4).

\section{The content of crude polysaccharides in stems}

Polysaccharides accumulation in stems was higher in all mycorrhizal seedlings than in the control without fungal inoculation (Fig. 5). Among the fungal strains tested, inoculation with the Sebacina strain S2 conferred the highest polysaccharides content.

\section{Discussion}

Most green orchids are known to associate with fungi from the polyphyletic rhizoctonia group, with its different clades such as Serendipitaceae (Sebacinales), Tulasnellaceae and Ceratobasidiaceae (Batty et al. 2006; Valadares et al. 2011). The mycorrhizal association of orchids could be compatible with several fungal groups or be highly specific for a narrow group of fungi (Otero et al. 2002; Ma et al. 2003; McCormick et al. 2004; Shefferson et al. 2005; Suárez et al. 2006). Fungus isolated from a peloton in adult orchid roots and its role as a seed germination symbiont cannot been ascertained until the results of symbiotic germination are known (Hoang et al. 2017). Furthermore, the non-compatible fungi may stimulate orchid seed germination, but they could not support subsequent seedling development (Rasmussen et al. 2015). In Dendrobium species, various fungal symbionts such as Cantharellaceae, Sebacinales and Tulasnellaceae are present in the protocorm and roots of adult plants (Chen et al. 2012). In the report by Tan et al. (2014), the ITS sequence of an efficient Tulasnella strain JC-02 in promoting seed germination of $D$. officinale is identical to Tulasnella strains S6 in this study, suggesting this efficient fungal strain may be dominant in wild Dendrobium populations. In previous reports, Sebacina strains could stimulate seed germination and further protocorm development of D. nobile and D. officinale (Wang et al. 2011; Zhao et al. 2013). In this study, the results of symbiotic germination revealed that $D$. officinale is compatible with different Tulasnella and Sebacina fungal strains for seed germination (Table 1; Fig. 3), although the efficiency of the strains differs. While Tulasnella strains S4 and S5 are not close related to Tulasnella strains S6 and S7 (Fig. 1), they could stimulate seed germination and protocorm

Table 1 Effect of fungal strains on seed germination and protocorm development of $D$. officinale at 15 weeks of inoculation

\begin{tabular}{|c|c|c|c|c|c|c|c|}
\hline \multirow[t]{2}{*}{ Treatment } & \multicolumn{6}{|c|}{ Ratio of seed germination and protocorm development $(\%)^{\mathbf{b}}$} & \multirow[t]{2}{*}{ Total germination $(\%)^{c}$} \\
\hline & Stage 0 & Stage 1 & Stage 2 & Stage 3 & Stage 4 & Stage 5 & \\
\hline Control $^{\mathrm{a}}$ & $25.72 \pm 2.85^{b}$ & $60.04 \pm 4.34^{\mathrm{a}}$ & $14.24 \pm 3.07^{d}$ & $0.00 \pm 0.00^{d}$ & $0.00 \pm 0.00^{b}$ & $0.00 \pm 0.00^{b}$ & $14.24 \pm 3.07^{d}$ \\
\hline $\mathrm{S} 2$ & $8.77 \pm 1.30^{c}$ & $6.56 \pm 3.95^{b c}$ & $25.97 \pm 5.41^{c}$ & $50.23 \pm 8.08^{a}$ & $8.48 \pm 4.00^{b}$ & $0.00 \pm 0.00^{b}$ & $84.67 \pm 8.85^{\mathrm{a}}$ \\
\hline S3 & $41.82 \pm 2.63^{a}$ & $9.23 \pm 0.67^{b c}$ & $14.86 \pm 0.90^{d}$ & $28.94 \pm 3.11^{b}$ & $5.14 \pm 2.16^{b}$ & $0.00 \pm 0.00^{b}$ & $48.95 \pm 4.02^{c}$ \\
\hline S4 & $12.87 \pm 10.01^{c}$ & $12.92 \pm 5.41^{b}$ & $40.86 \pm 8.43^{b}$ & $28.53 \pm 2.59^{b c}$ & $4.69 \pm 0.83^{b}$ & $0.00 \pm 0.00^{b}$ & $74.08 \pm 7.31^{b}$ \\
\hline S5 & $9.15 \pm 1.95^{c}$ & $8.01 \pm 1.24^{b c}$ & $64.20 \pm 2.73^{a}$ & $18.80 \pm 1.39^{c}$ & $0.00 \pm 0.00^{b}$ & $0.00 \pm 0.00^{b}$ & $83.00 \pm 1.39^{a b}$ \\
\hline S6 & $5.47 \pm 1.96^{c}$ & $10.40 \pm 5.98^{b}$ & $37.43 \pm 7.03^{b}$ & $23.60 \pm 8.49^{b c}$ & $19.97 \pm 6.49^{\mathrm{a}}$ & $1.35 \pm 1.09^{b}$ & $86.35 \pm 3.07^{\mathrm{a}}$ \\
\hline S7 & $5.10 \pm 2.60^{c}$ & $1.83 \pm 2.28^{c}$ & $7.08 \pm 6.58^{d}$ & $7.84 \pm 3.56^{d}$ & $19.20 \pm 9.80^{a}$ & $59.17 \pm 8.78^{a}$ & $93.29 \pm 4.20^{\mathrm{a}}$ \\
\hline
\end{tabular}

a Control, the seeds without fungal inoculation

${ }^{b}$ Germination percentages (mean $\pm \mathrm{SE}, \mathrm{n}=3$ ) within columns marked by different letters are significantly different at $P<0.05$ (Fisher's protected least significant difference test)

c Germination was defined as emergence of the embryo from the seed coat (stage 2) 

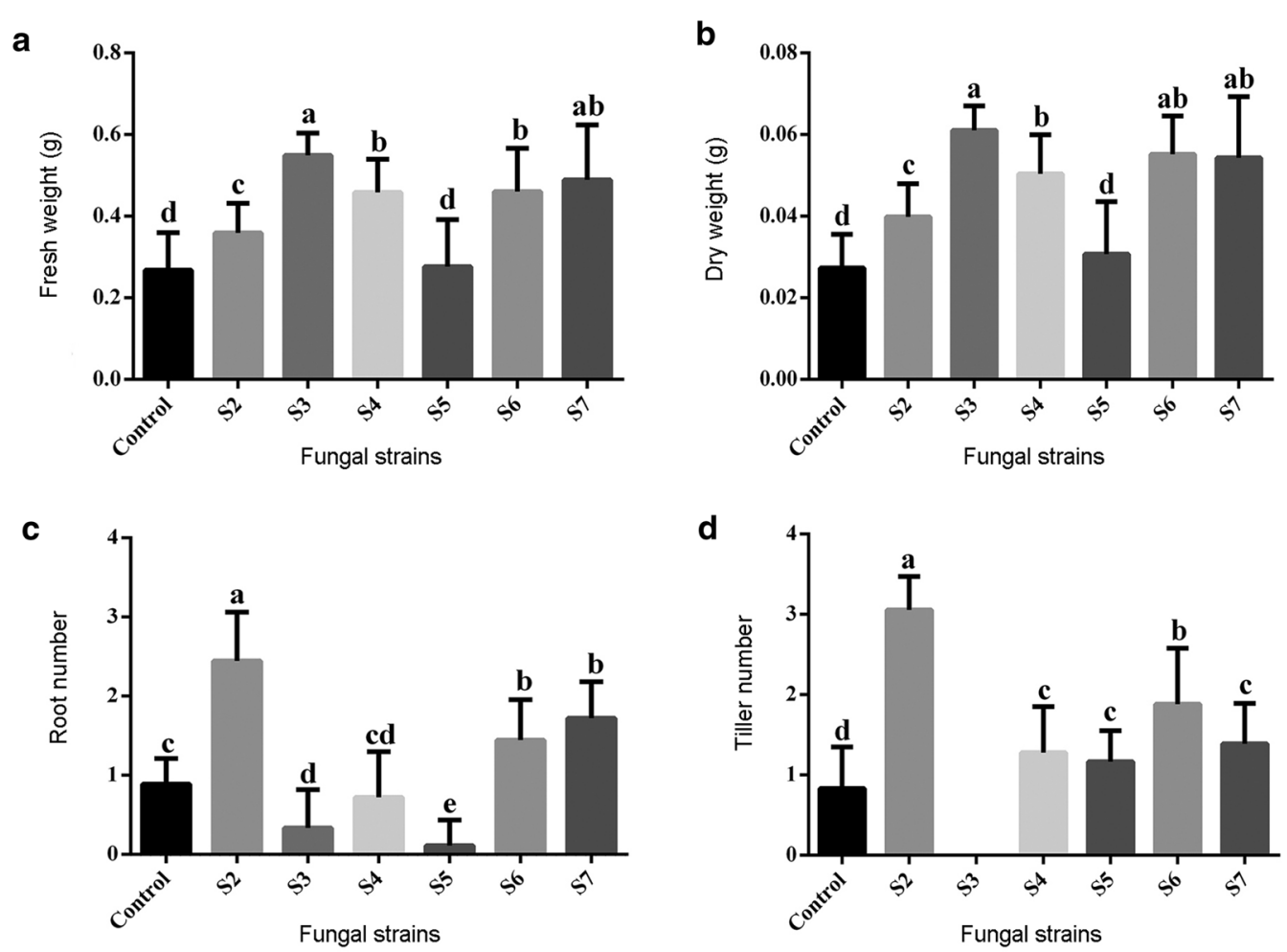

Fig. 4 Effect of fungal strains on seedling growth of $D$. officinale at 15 weeks after inoculation. Change in a fresh weight, $\mathbf{b}$ dry weight, c root number, and $\mathbf{d}$ tiller number. Data are mean \pm SE. Different letters above bars are significantly different at $P<0.05$ (Fisher's protected least significant difference test)

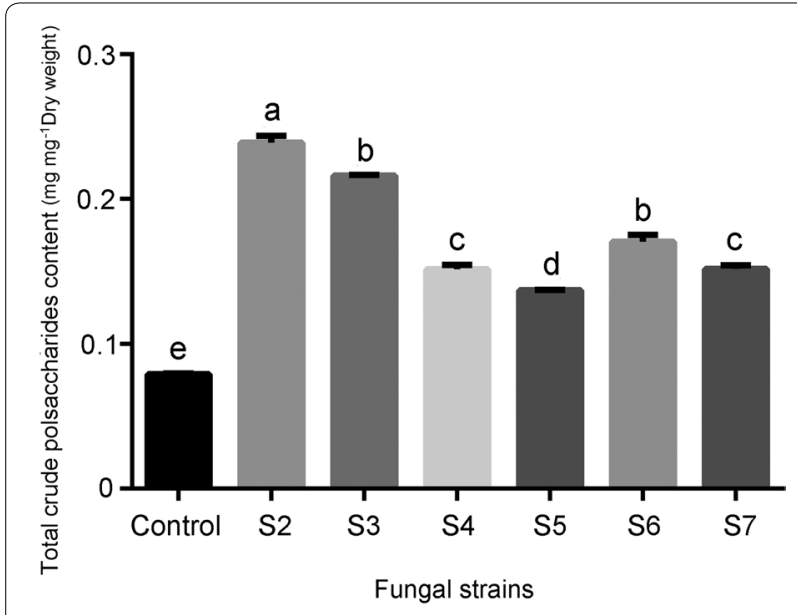

Fig. 5 Effect of fungal strains on total crude polysaccharides content in stems of $D$. officinale at 15 weeks after inoculation. Data are mean \pm SE. Different letters above bars are significantly different at $P<0.05$ (Fisher's protected least significant difference test)

formation to stage 2 or 3 after 15 weeks of inoculation. Although they are not as effective as Tulasnella strains S6 and S7, a few seedlings were observed by 30 weeks of inoculation (data not shown). Our data suggest a nonspecific fungal association (at least two different fungal clades, i.e. Sebacinales and Tulasnellaceae, Fig. 1) during germination in $D$. officinale.

In the present study, the mycobionts strains tested had different effects on growth and development of seedlings (Fig. 4). Although Sebacina strain S3 had little effect on seed germination, it significantly improved the growth of seedlings (i.e., optimal fresh and dry matter yield) (Figs. 4a, b). In orchids, mycorrhizal symbionts may switch in different developmental stages (McCormick et al. 2004; Rafter et al. 2016). Previous studies have demonstrated that compatible fungi for promoting seed germination may not be able to support subsequent seedling development (Bidartondo and Read 2008; Huynh et al. 2009; Rasmussen et al. 2015). Inoculation with Sebacina strain S2 greatly improved the number of roots and tillers of seedlings (Fig. 4c, d) but had little effect on yield of fresh and dry matter (Fig. 4a, b). The changes in plant growth pattern (i.e., dwarfism, multiple shoots and roots) after inoculation with Sebacina strain S2 may be attributed to hormonal compounds derived from mycorrhizal fungus. The production of plant hormones by symbiotic 
fungi can affect the growth and development of host plants (Hirsch et al. 1997). Further research into plant hormones produced by mycorrhizal fungi would provide insights into the growth and development of Dendrobium seedlings in symbiotic cultures.

Both Sebacina strains S2 and S3 were unable to support post-germination development beyond stage 3 by 15 weeks of inoculation (Table 1). In adult orchids, different fungal strains could be isolated from the same single peloton or from the same single root (Kristiansen et al. 2001; Raleigh et al. 2001; McKendrick et al. 2002; Taylor et al. 2003; Bougoure et al. 2005). Under natural conditions, the broadening and/ or switch of a mycorrhizal association may enable orchids to adapt to the varied physiological changes during seedling development, including the switch to partial or full autotrophy, an increase in transpiration, or environmental fluctuations (Těšitelová et al. 2012).

The crude polysaccharides, the non-starch, hetero-polysaccharides, have been considered the main indicative ingredients in medicinal Dendrobium species (Meng et al. 2013; Dave and Shah 2015). Our data show a significant effect on the accumulation of crude polysaccharides in mycorrhizal seedlings of $D$. officinale as compared with the control (Fig. 5). Increased crude polysaccharides content has been reported in seedlings of $D$. nobile after fungal inoculation (Li et al. 2017). Moreover, we found a diverse effect on the growth and crude polysaccharides content of seedlings with different fungal treatments. Although conferring less fresh and dry matter yield, inoculation with Sebacina strain S2 resulted in the highest crude polysaccharides content as compared with other Sebacina and Tulasnella strains. Seedlings inoculated with Sebacina strain S3 had optimal fresh and dry matter yield and also relatively high crude polysaccharides content (about 21.6\% less than S2). Fungal elicitors were found to affect the accumulation of active ingredients in medical plants by changing the expression of specific genes involved in secondary metabolite biosynthesis (Zhai et al. 2017).

Tulasnella strains S6 or S7 could stimulate seed germination and also support the growth of seedlings (Fig. 4a, b). For Drakaea, a single mycorrhizal symbiont could support growth from the protocorm to seedling and adult stages (Phillips et al. 2011). In the conservation or production practices of $D$. officinale, inoculation with Tulasnella strains S6 or S7 for all developing stages may simplify the operation. In terms of medicinal plant production, the addition of Sebacina strains S2 or S3 to young seedlings may increase crude polysaccharides content or fresh and dry matter yield.

\section{Conclusions}

The present study demonstrates that for symbiotic germination, D. officinale is compatible with Tulasnella and Sebacina strains, thereby suggesting a non-specific mycorrhizal association. Seedlings inoculated with Sebacina strains S2 and S3 showed optimal crude polysaccharides content and fresh and dry matter yield, respectively, although with little effect on seed germination. These fungal strains tested in symbiotic cultures have different effects on growth and development of seedlings, which suggests development-dependent specificity. Our data provide basic knowledge of use of different fungal strains in conservation and/or production practices.

\section{Supplementary information}

Supplementary information accompanies this paper at https://doi. org/10.1186/s40529-019-0278-6.

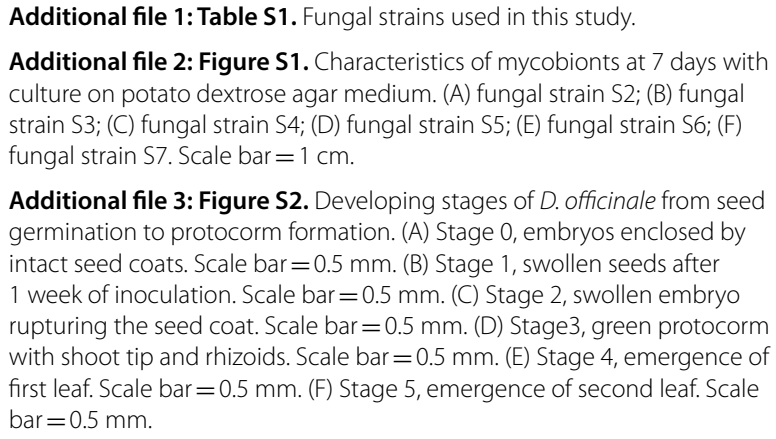

Additional file 3: Figure S2. Developing stages of D. officinale from seed germination to protocorm formation. (A) Stage 0, embryos enclosed by intact seed coats. Scale bar $=0.5 \mathrm{~mm}$. (B) Stage 1, swollen seeds after 1 week of inoculation. Scale bar $=0.5 \mathrm{~mm}$. (C) Stage 2, swollen embryo rupturing the seed coat. Scale bar $=0.5 \mathrm{~mm}$. (D) Stage3, green protocorm with shoot tip and rhizoids. Scale bar $=0.5 \mathrm{~mm}$. (E) Stage 4, emergence of first leaf. Scale bar $=0.5 \mathrm{~mm}$. (F) Stage 5, emergence of second leaf. Scale bar $=0.5 \mathrm{~mm}$.

\section{Acknowledgements}

This work was supported by grants from the Natural Science Foundation of China (81573526) to GSX.

\section{Authors' contributions}

LYI and GSX conceived the study. LYI, GSX, LYY and ZY designed the study. GSX and LYI provided funding. ZY and LYY performed experiments. LYI, GSX and ZY wrote the paper. All authors read and approved the final manuscript.

\section{Funding}

This work was supported by Grants from the Natural Science Foundation of China (81573526) to GSX. The funder had no role in the design of the study; the collection, analyses, or interpretation of data; the writing of the manuscript, or the decision to publish. Funding was funded by the Innovation Fund for Medical Sciences Grant (2017-I2M-3-013).

Availability of data and materials

Not applicable.

Ethics approval and consent to participate

Not applicable.

Consent for publication

Not applicable.

Competing interests

The authors declare that they have no competing interests. 


\section{Author details}

${ }^{1}$ Institute of Medicinal Plant Development, Chinese Academy of Medical Sciences \& Peking Union Medical College, Beijing 100193, People's Republic of China. ${ }^{2}$ Biology Department, National Museum of Natural Science, Taichung 40453, Taiwan. ${ }^{3}$ Department of Life Sciences, National Chung Hsing University, Taichung 40227, Taiwan.

Received: 12 October 2019 Accepted: 24 December 2019 Published online: 27 January 2020

\section{References}

Barrett CF, Freudenstein JV, Taylor DL, Kõljalg U (2010) Range wide analysis of fungal associations in the fully mycoheterotrophic Corallorhiza striata complex (Orchidaceae) reveals extreme specificity on ectomycorrhizal Tomentella (Thelephoraceae) across North America. Am J Bot 97:628-643

Batty AL, Brundrett MC, Dixon KW, Sivasithamparam K (2006) New methods to improve symbiotic propagation of temperate terrestrial orchid seedlings from axenic culture to soil. Aust J Bot 54:367-374

Bidartondo MI (2005) The evolutionary ecology of mycoheterotrophy. New Phytol 167:335-352

Bidartondo MI, Read DJ (2008) Fungal specificity bottlenecks during orchid germination and development. Mol Ecol 17:3707-3716

Bougoure JJ, Bougoure DS, Cairney JW, Dearnaley JD (2005) ITS-RFLP and sequence analysis of endophytes from Acianthus, Caladenia and Pterostylis (Orchidaceae) in southeastern Queensland. Mycol Res 109:452-460

Chen J, Wang H, Guo SX (2012) Isolation and identification of endophytic and mycorrhizal fungi from seeds and roots of Dendrobium (Orchidaceae). Mycorrhiza 22:297-307

Dave DT, Shah GB (2015) Pharmacological potential of naturally occurring non-starch polysaccharides (NSP). J Phytopharmacol 4:307-310

Dearnaley JD, Cameron DD (2017) Nitrogen transport in the orchid mycorrhizal symbiosis-further evidence for a mutualistic association. New Phytol 213:10-12

Dearnaley JDW, Martos F, Selosse MA (2012) Orchid mycorrhizas: molecular ecology, physiology, evolution and conservation aspects. In: Hock B (ed) Fungal association. Springer, Berlin

Felsenstein J (1985) Confidence limits on phylogenies: an approach using the bootstrap. Evolution 39:783-791

Fochi V, Chitarra W, Kohler A, Voyron S, Singan VR, Lindquist EA, Barry KW, Girlanda M, Grigoriev IV, Martin F, Balestrini R, Perotto S (2017) Fungal and plant gene expression in the Tulasnella calospora-Serapias vomeracea symbiosis provides clues about nitrogen pathways in orchid mycorrhizas. New Phytol 213:365-379

Gardes M, Bruns TD (1993) ITS primers with enhanced specificity for basidiomycetes-application to the identification of mycorrhizae and rusts. Mol Ecol 2:113-118

Hirsch AM, Fang Y, Asad S, Kapulnik Y (1997) The role of phytohormones in plant-microbe symbioses. Plant Soil 194:171-184

Hoang NH, Kane ME, Radcliffe EN, Zettler LW, Richardson LW (2017) Comparative seed germination and seedling development of the ghost orchid, Dendrophylax lindenii (Orchidaceae), and molecular identification of its mycorrhizal fungus from South Florida. Ann Bot 119:379-393

Huynh TT, Thomson R, Mclean CB, Lawrie AC (2009) Functional and genetic diversity of mycorrhizal fungi from single plants of Caladenia formosa (Orchidaceae). Ann Bot 104:757-765

Kennedy AH, Tatlor DL, Watson LE (2011) Mycorrhizal specificity in the fully mycoheterotrophic Hexalectris Raf. (Orchidaceae: Epidendroideae). Mol Ecol 20:1303-1316

Kristiansen KA, Rasmussen FN, Rasmussen HN (2001) Seedlings of Neuwiedia (Orchidaceae subfamily Apostasioideae) have typical orchidaceous mycotrophic protocorms. Am J Bot 88:956-959

Kuga U, Sakamoto N, Yurimoto H (2014) Stable isotope imaging reveals that both live and degenerating fungal pelotons transfer carbon and nitrogen to orchid protocorms. New Phytol 202:594-605

Leake JR (1994) The biology of myco-heterotrophic ('saprophytic') plants. New Phytol 127:171-216

Li Q, Li B, Zhou LS, Ding G, Li B, Guo SX (2017) Molecular analysis of polysaccharide accumulation in Dendrobium nobile infected with the mycorrhizal fungus Mycena sp. RSC Advances 7:25872-25884
Li YY, Chen XM, Zhang Y, Cho YH, Wang AR, Yeung EC, Zeng X, Guo SX, Lee YI (2018a) Immunolocalization and changes of hydroxyproline-rich glycoproteins during symbiotic germination of Dendrobium officinale. Front Plant Sci 9:552

Li YY, Wang H, Guo SX (2018b) Protocols for establishing fungi-protocorm culture. In: Lee YI, Yeung ECT (eds) Orchid propagation: from laboratories to greenhouses - methods and protocols. Springer protocols handbooks. Humana Press, New York

Ma M, Tan TK, Wong SM (2003) Identification and molecular phylogeny of Epulorhiza isolates from tropical orchids. Mycol Res 107:1041-1049

McCormick MK, Whigham DF, O'Neill J (2004) Mycorrhizal diversity in photosynthetic terrestrial orchids. New Phytol 163:425-438

McKendrick SL, Leake JR, Taylor DL, Read DJ (2002) Symbiotic germination and development of the myco-heterotrophic orchid Neottia nidus-avis in nature and its requirement for locally distributed Sebacina spp. New Phytol 154:233-247

Meng LZ, Lv GP, Hu DJ, Cheong KL, Xie J, Zhao J, Li SP (2013) Effects of polysaccharides from different species of Dendrobium (Shihu) on macrophage function. Molecules 18:5779-5791

Murashige T, Skoog F (1962) A revised medium for rapid growth and bioassays with tobacco tissue cultures. Physiol Plant 15:473-497

Ogura-Tsujita Y, Yokoyama J, Miyoshi K, Yukawa T (2012) Shifts in mycorrhizal fungi during the evolution of autotrophy to mycoheterotrophy in Cymbidium (Orchidaceae). Am J Bot 99:1158-1176

Otero JT, Ackerman JD, Bayman P (2002) Diversity and host specificity of endophytic Rhizoctonia-like fungi from tropical orchids. Am J Bot 89:1852-1858

Pharmacopoeia Committee of the P. R. China (2005) Pharmacopoeia of the People's Republic of China, vol 1. People's Medical Publishing House, Beijing

Phillips RD, Brown AP, Dixon KW, Hopper SD (2011) Orchid biogeography and factors associated with rarity in a biodiversity hotspot, the Southwest Australian floristic region. J Biogeogr 38:487-501

Porras-Alfaro A, Bayman P (2007) Mycorrhizal fungal of Vanilla: diversity, specificity and effects on seed germination and plant growth. Mycologla 99:510-525

Rafter M, Yokoya K, Schofield EJ, Zettler LW, Sarasan V (2016) Non-specific symbiotic germination of Cynorkis purpurea (Thouars) Kraezl., a habitatspecific terrestrial orchid from the Central Highlands of Madagascar. Mycorrhiza 26:541-552

Raleigh RE, Cross RG, Lawrie AC, Coates F, Moorrees ACA (2001) Research into the propagation of eastern Australian Caladenia. In: Barrett RL, Dixon KW (eds) Book of extended abstracts: proceedings from the first international Orchid conservation congress. Plant Conservation Association Inc., London, p 115

Rasmussen HN (1995) Terrestrial orchids-from seed to mycotrophic plant. Cambridge University Press, Cambridge

Rasmussen HN, Dixon KW, Jersáková J, Těšitelová T (2015) Germination and seedling establishment in orchids: a complex of requirements. Ann Bot 116:391-402

Saitou N, Nei M (1987) The neighbor-joining method: a new method for reconstructing phylogenetic trees. Mol Biol Evol 4:406-425

Shefferson RP, Weiß M, Kull T, Taylor DL (2005) High specificity generally characterizes mycorrhizal association in rare lady's slipper orchids, genus Cypripedium. Mol Ecol 14:613-626

Shefferson RP, Taylor DL, Weiss M, Garnica S, McCormick MK, Adams S, Gray HM, McFarland JW, Kull T, Tali K, Yukawa T, Kawahara T, Miyoshi K, Lee YI (2007) The evolutionary history of mycorrhizal specificity among lady's slipper orchids. Evolution 61:1380-1390

Smith SE, Read DJ (2008) Mycorrhizal symbiosis, 3rd edn. Academic Press, San Diego

Stewart SL, Zettler LW, Minso J, Brown PW (2003) Symbiotic germination and reintroduction of Spiranthes brevilabris Lindley, an endangered orchid native to Florida. Selbyana 24:64-70

Suárez JP, Weiß M, Abele A, Garnica S, Oberwinkler F, Kottke I (2006) Diverse tulasnelloid fungi form mycorrhizas with epiphytic orchids in an Andean cloud forest. Mycol Res 110:1257-1270

Tan XM, Wang CL, Chen XM, Zhou YQ, Wang YQ, Luo AX, Liu ZH, Guo SX (2014) In vitro seed germination and seedling growth of an endangered epiphytic orchid, Dendrobium officinale, endemic to China using mycorrhizal fungi (Tulasnella sp.). Sci Hortic 165:62-68 
Taylor DL, Bruns TD, Szaro TM, Hodges SA (2003) Divergence in mycorrhizal specialization within Hexalectris spicata (Orchidaceae), a nonphotosynthetic desert orchid. Am J Bot 90:1168-1179

Těšitelová T, Těšitel J, Jersáková J, Říhová G, Selosse MA (2012) Symbiotic germination capability of four Epipactis species (Orchidaceae) is broader than expected from adult ecology. Am J Bot 99:1020-1032

Valadares R, Pereira M, Otero J, Cardoso E (2011) Narrow fungal mycorrhizal diversity in a population of the orchid Coppensia doniana. Biotropica 44:114-122

Wang H, Fang HY, Wang YQ, Duan LS, Guo SX (2011) In situ seed baiting techniques in Dendrobium officinale Kimura et Migo and Dendrobium nobile

Lindl.: the endangered Chinese endemic Dendrobium (Orchidaceae). World J Microbiol Biotechnol 27:2051-2059

Wang X, Li Y, Song X, Meng Q, Zhu J, Zhao Y, Yu W (2017) Influence of host tree species on isolation and communities of mycorrhizal and endophytic fungi from roots of a tropical epiphytic orchid, Dendrobium sinense (Orchidaceae). Mycorrhiza 27:709-718

Wang DH, Fan B, Wang Y, Zhang LJ, Wang FZ (2018) Optimum extraction, characterization, and antioxidant activities of polysaccharides from flowers of Dendrobium devonianum. Int J Anal Chem 2018:1-8

White TJ, Bruns T, Lee S, Taylor J (1990) Amplification and direct sequencing of fungal ribosomal RNA genes for phylogenetics. In: Innis MA, Gelfand DH, Sninsky JJ, White T (eds) PCR Protocols: a guide to methods and applications. Academic Press, San Diego
Xu JT, Mu C (1990) The relation between growth of Gastrodia elata protocorms and fungi. Acta Bot Sinica 32:26-31 (In Chinese)

Yeung EC, Chan CKW (2015) The glycol methacrylate embedding resins-Technovit 7100 and 8100. Plant microtechniques and protocols. Springer, Berlin, pp 67-82

Zhai X, Jia M, Chen L, Zheng CJ, Rahman K, Han T, Qin LP (2017) The regulatory mechanism of fungal elicitor-induced secondary metabolite biosynthesis in medical plants. Crit Rev Microbiol 43:238-261

Zhao MM, Zhang G, Zhang DW, Hsiao YY, Guo SX (2013) ESTs analysis reveals putative genes involved in symbiotic seed germination in Dendrobium officinale. PLOS ONE 8:e72705

Zi MX, Sheng CL, Goodale UM, Shao SC, Ga JY (2014) In situ seed baiting to isolate germination-enhancing fungi for an epiphytic orchid, Dendrobium aphyllum (Orchidaceae). Mycorrhiza 24:487-499

\section{Publisher's Note}

Springer Nature remains neutral with regard to jurisdictional claims in published maps and institutional affiliations.

\section{Submit your manuscript to a SpringerOpen ${ }^{\circ}$ journal and benefit from:}

- Convenient online submission

- Rigorous peer review

- Open access: articles freely available online

- High visibility within the field

- Retaining the copyright to your article

Submit your next manuscript at $\boldsymbol{\nabla}$ springeropen.com 